\title{
Increased T-helper 17.1 cells in sarcoidosis mediastinal lymph nodes
}

\author{
Caroline E. Broos ${ }^{1}$, Laura L. Koth², Menno van Nimwegen', \\ Johannes C.C.M. in 't Veen ${ }^{3}$, Sandra M.J. Paulissen", Jan Piet van Hamburg ${ }^{5}$, \\ Jouke T. Annema ${ }^{6}$, Roxane Heller-Baan ${ }^{7}$, Alex Kleinjan ${ }^{1}$, Henk C. Hoogsteden ${ }^{1}$, \\ Marlies S. Wijsenbeek ${ }^{1}$, Rudi W. Hendriks ${ }^{1}$, Bernt van den Blink ${ }^{1,8}$ and \\ Mirjam Kool ${ }^{1,8}$
}

\begin{abstract}
Affiliations: 'Dept of Pulmonary Medicine, Erasmus MC, Rotterdam, The Netherlands. ${ }^{2}$ Dept of Medicine, Division of Pulmonary and Critical Care, University of California, San Francisco, CA, USA. ${ }^{3}$ Dept of Pulmonology, Franciscus Gasthuis \& Vlietland, Rotterdam, The Netherlands. ${ }^{4}$ Dept of Rheumatology, Erasmus MC, Rotterdam, The Netherlands. ${ }^{5}$ Dept of Experimental Immunology, Amsterdam Rheumatology and Immunology Center, Academic Medical Center, University of Amsterdam, Amsterdam, The Netherlands. ${ }^{6}$ Dept of Pulmonology, Academic Medical Center, University of Amsterdam, Amsterdam, The Netherlands. ${ }^{7}$ Dept of Pulmonology, Ikazia Hospital, Rotterdam, The Netherlands. ${ }^{8}$ These two authors are joint senior authors.
\end{abstract}

Correspondence: Mirjam Kool, Dept of Pulmonary Medicine, Erasmus MC, 's-Gravendijkwal 230, 3015 CE, Rotterdam, The Netherlands. E-mail: m.koolderasmusmc.nl

@ERSpublications

Th17-lineage cells are increased in sarcoidosis MLNs and BALF Th17.1 cell proportions correlate with disease progression http://ow.ly/hBb730hSEv7

Cite this article as: Broos CE, Koth LL, van Nimwegen M, et al. Increased T-helper 17.1 cells in sarcoidosis mediastinal lymph nodes. Eur Respir J 2018; 51: 1701124 [https://doi.org/10.1183/ 13993003.01124-2017].

ABSTRACT The lung-draining mediastinal lymph nodes (MLNs) are currently widely used to diagnose sarcoidosis. We previously reported that T-helper (Th) 17.1 cells are responsible for the exaggerated interferon- $\gamma$ production in sarcoidosis lungs. In this study, we aimed to investigate 1) whether Th17.1 cells are also increased in the MLNs of sarcoidosis patients and 2) whether frequencies of the Th17.1 cells at diagnosis may correlate with disease progression.

MLN cells from treatment-naive pulmonary sarcoidosis patients $(n=17)$ and healthy controls $(n=22)$ and peripheral blood mononuclear cells $(n=34)$ and bronchoalveolar lavage fluid (BALF) $(n=36)$ from sarcoidosis patients were examined for $\mathrm{CD}^{+} \mathrm{T}$-cell subset proportions using flow cytometry.

Higher proportions of Th17.1 cells were detected in sarcoidosis MLNs than in control MLNs. Higher Th17.1 cell proportions were found in sarcoidosis BALF compared with MLNs and peripheral blood. Furthermore, BALF Th17.1 cell proportions were significantly higher in patients developing chronic disease than in patients undergoing resolution within 2 years of clinical follow-up.

These data suggest that Th17.1 cell proportions in pulmonary sarcoidosis can be evaluated as a diagnostic and/or prognostic marker in clinical practice and could serve as a new therapeutic target.

This article has supplementary material available from erj.ersjournals.com

Received: Nov 242016 | Accepted after revision: Jan 022018

Support statement: M. Kool was supported by a FP7 Marie Curie CIG grant. C.E. Broos was supported by a Travel Award from the ATS PAR member Foundation for Sarcoidosis Research. Funding information for this article has been deposited with the Crossref Funder Registry.

Conflict of interest: C.E. Broos, R.W. Hendriks, M. Kool have a patent P117584NL00 (Th17.1 cells as biomarker for sarcoidosis disease prognosis) pending to C.E. Broos, R.W. Hendriks, M. Kool and J.R. Miedema.

Copyright @ERS 2018 


\section{Introduction}

Sarcoidosis is a multisystem granulomatous disorder of unknown cause, predominantly affecting the lungs [1]. The disease affects people all over the world, but the incidence/prevalence rates and natural course of the disease vary greatly [1-3]. Intriguingly, the disease resolves in the majority of sarcoidosis patients, but granulomas persist in $\sim 30 \%$ of patients, which can lead to chronic, sometimes progressive disease with need for treatment [1, 4]. It still remains largely unknown what environmental, genetic and/or immunological factors determine the disease course of (non-Löfgren) sarcoidosis patients. Consequently, no variables have yet been identified that are routinely used in clinical practice to help predict the disease course of sarcoidosis patients.

We have recently reported that the exaggerated interferon (IFN)- $\gamma$ in sarcoidosis lungs is predominantly derived from T-helper (Th) 17.1 cells rather than from Th1 cells [5]. One of the cardinal features of pulmonary sarcoidosis is mediastinal lymphadenopathy. The lung-draining mediastinal lymph nodes (MLNs) have become an attractive clinical site for sarcoidosis diagnosis as detection of granulomas is more accurate in MLNs than in lung [6]. Given the extent of MLN involvement and its strong diagnostic value in pulmonary sarcoidosis, we aimed to investigate 1) whether Th17.1 cells are also increased in the MLNs of sarcoidosis patients (which could have added value for the diagnosis) and 2) whether frequencies of the Th17.1 cells at diagnosis may correlate with disease progression after 2 years. Therefore, in the current study we investigated the distributions of $\mathrm{CD}^{+}$T-cell subsets in control and sarcoidosis MLNs, and compared them to sarcoidosis peripheral blood and bronchoalveolar lavage fluid (BALF).

\section{Materials and methods}

\section{Study design and subjects}

Patients with pulmonary sarcoidosis were included at time of diagnosis. The diagnosis of sarcoidosis was made according to the guidelines of the American Thoracic Society/European Respiratory Society/World Association of Sarcoidosis and Other Granulomatous Disorders [1].

Exclusion criteria were use of immunomodulatory medication 3 months prior to study inclusion, respiratory tract infection 4 weeks prior to study inclusion, and concomitant pulmonary disease (including chronic obstructive pulmonary disorder and asthma), autoimmune diseases, malignancies, HIV seropositivity, pregnancy and allergies.

For this study, 55 sarcoidosis patients donated BALF, MLN-derived fine needle aspiration or peripheral blood. Supplementary figure E1 shows a detailed flowchart describing the material each participating patient donated for this study.

Disease course of a subgroup ( $\mathrm{n}=25$ with data available) of patients was determined 2 years after study inclusion. Resolution of disease was defined by the absence of abnormalities on chest radiography and clinical symptoms. Patients with residual abnormalities on chest radiography but without need for treatment were designated as nonprogressive chronic and patients with need for treatment were designated as progressive chronic.

Control MLNs were collected from 22 lung transplantation donors without signs of pulmonary inflammation (routinely assessed by chest radiography and bronchoscopy).

The Medical Ethical Committee of the Erasmus MC (Rotterdam, The Netherlands) approved this study. Written informed consent was obtained from every participant before study inclusion. Further subject characteristics are shown in table 1.

\section{Study materials}

Bronchoscopy with bronchoalveolar lavage was performed as previously described [7]. BALF cells and peripheral blood were processed as previously described [7].

Endoscopic or endobronchial ultrasound-guided fine needle aspirations from draining MLNs were performed with a $22 \mathrm{G}$ (or 19G) needle. MLN aspirates were filtered through a $100 \mu \mathrm{m}$ cell strainer (BD Biosciences, Oxford, UK) and centrifuged. Cells were stored at $-150^{\circ} \mathrm{C}$.

Control MLNs were collected from lung transplantation donors. Control MLNs were processed as lymph node aspirates.

\section{Flow cytometry}

BALF, MLN and peripheral blood mononuclear cells were stained for intra- and extracellular markers using the antibodies listed in supplementary table E1. Th subsets of CD45RA ${ }^{-}$FoxP $3^{\text {low/int }}$ total memory Th cells were identified on the basis of chemokine receptor expression (supplementary figure E2). Fixable Aqua Dead Cell Stain kit for 405 nm (ThermoFisher, Paisley, UK) was used as live/dead marker. At least 
100000 cells per sample were measured on a flow cytometer LSRII (BD Biosciences), and the mean fluorescence intensity of cytotoxic T-lymphocyte antigen 4 (CTLA4) was standardised to average expression in healthy control peripheral blood cells, stated as change in mean fluorescence intensity. Ki-67 staining was used to assess the proliferative status of cell subsets.

\section{Statistical analyses}

Comparisons were performed using the Mann-Whitney U-test or Wilcoxon signed-rank test. p-values were two-sided and analyses were performed using SPSS Statistics version 21 (IBM, Armonk, NY, USA). $\mathrm{p}<0.05$ was considered statistically significant.

\section{Results}

Significant increase of Th17.1 cells in sarcoidosis MLNs compared with controls

We previously found an increased presence of IFN $-\gamma^{+}$Th17.1 cells in sarcoidosis BALF compared with controls [5]. We questioned whether Th17.1 cells are also increased in sarcoidosis MLNs compared with controls. As Th17.1 cells are primarily thought to be progeny of Th17 cells [8-15], we aimed to quantify Th1, Th2 and all CCR6 ${ }^{+}$Th subpopulations in sarcoidosis BALF $(\mathrm{n}=36)$, sarcoidosis MLNs $(\mathrm{n}=17)$ and control MLNs $(n=22)$ (for gating strategy, see supplementary figure E3). The CCR6 ${ }^{+}$double-positive cells are thought to reflect an intermediate stage between Th17 and Th17.1 cells [16-18].

In sarcoidosis BALF, Th17.1 cell proportions were higher than either Th1, Th2, Th17 or CCR6 ${ }^{+}$ double-positive cells (figure 1a) and thus represented the most predominant Th population in BALF (figure 1b). Similar to sarcoidosis BALF [5], sarcoidosis MLNs contained higher proportions of CCR6 ${ }^{+}$Th cells than control MLNs (figure 1c). Importantly, we identified significantly increased proportions of Th17.1 cells in sarcoidosis MLNs compared with control MLNs (figure 1d). Furthermore, sarcoidosis MLNs contained increased proportions of Th17.1 precursor cells, i.e. Th17 [19] and CCR6 double-positive cells, compared with controls (figure 1d and e). In sarcoidosis MLNs, Th1 cell proportions

\section{TABLE 1 Study subject characteristics}

\begin{tabular}{|c|c|c|}
\hline & Control & Sarcoidosis \\
\hline Subjects & 22 & 55 \\
\hline Age years & $47(15-70)$ & $43(24-75)$ \\
\hline Male/female & $6 / 16$ & $36 / 19$ \\
\hline \multicolumn{3}{|l|}{ Ethnicity } \\
\hline White & & $38(69)$ \\
\hline Black & & $8(15)$ \\
\hline Other & & $5(9)$ \\
\hline Unknown & & $4(7)$ \\
\hline \multicolumn{3}{|l|}{ Scadding stage } \\
\hline 0 & & $5(9)^{\#}$ \\
\hline I & & $20(36)$ \\
\hline II & & $23(42)$ \\
\hline III & & $4(7)$ \\
\hline Unknown & & $3(6)^{\pi}$ \\
\hline \multicolumn{3}{|l|}{ Diagnosis assessed by } \\
\hline TBB-EBB & & $17(31)$ \\
\hline BALF CD4/CD8 >3.5 & & $8(15)$ \\
\hline$E(B) U S-F N A$ & & $26(47)$ \\
\hline Other (e.g. mediastinoscopy, other biopsy) & & $4(7)$ \\
\hline \multicolumn{3}{|l|}{ Extrathoracic involvement } \\
\hline No/yes & & $21 / 34$ \\
\hline Skin & & $8(15)$ \\
\hline Eyes & & $16(29)$ \\
\hline Articular ${ }^{+}$ & & $22(40)$ \\
\hline Central nervous system & & $3(6)$ \\
\hline Other & & $6(11)$ \\
\hline
\end{tabular}

Data are presented as n, median (range) or $\mathrm{n}(\%)$. TBB: transbronchial biopsy; EBB: endobronchial biopsy; BALF: bronchoalveolar lavage fluid; E(B)US-FNA: endoscopic (endobronchial) ultrasound-guided fine needle aspiration. ${ }^{\#}$ : three patients had stage I and two patients had stage II sarcoidosis, determined by computed tomography (CT) scan; ": two patients had stage I and one patient had stage II sarcoidosis, determined by CT scan; ${ }^{+}$: self-reported articular involvement. 
a)

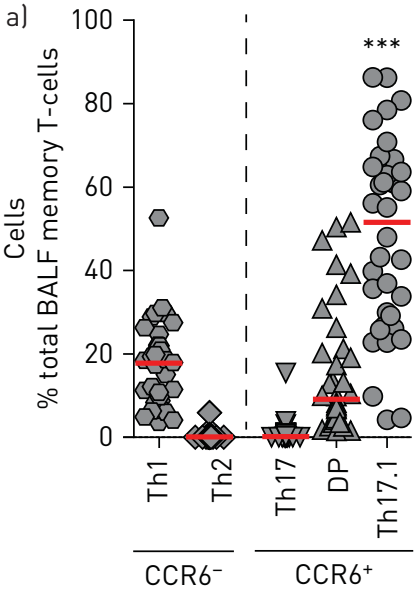

c)

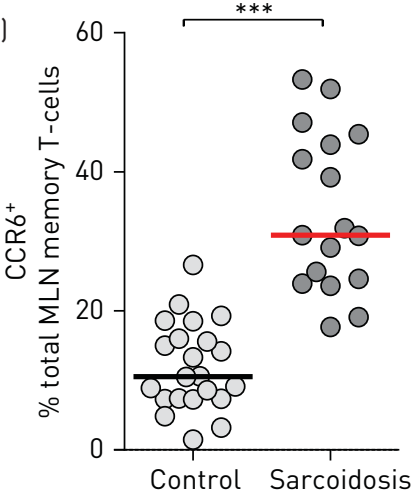

e)

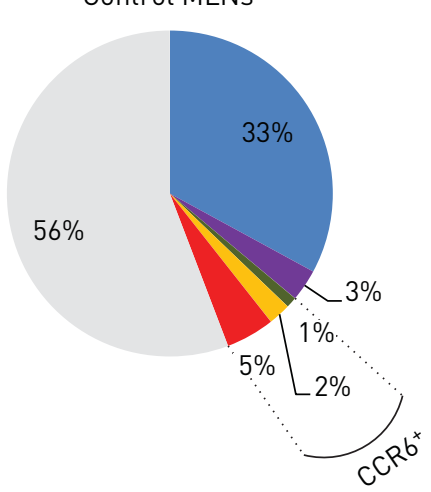

b)

Sarcoidosis BALF

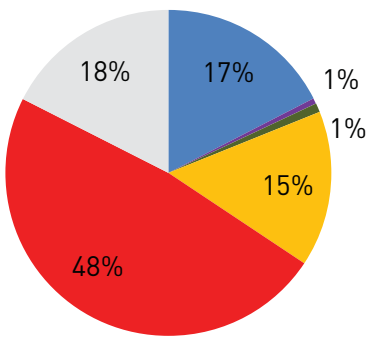

- Th1

- Th2

- Th17

$\square \mathrm{DP}$

- Th17.1

Unclassified

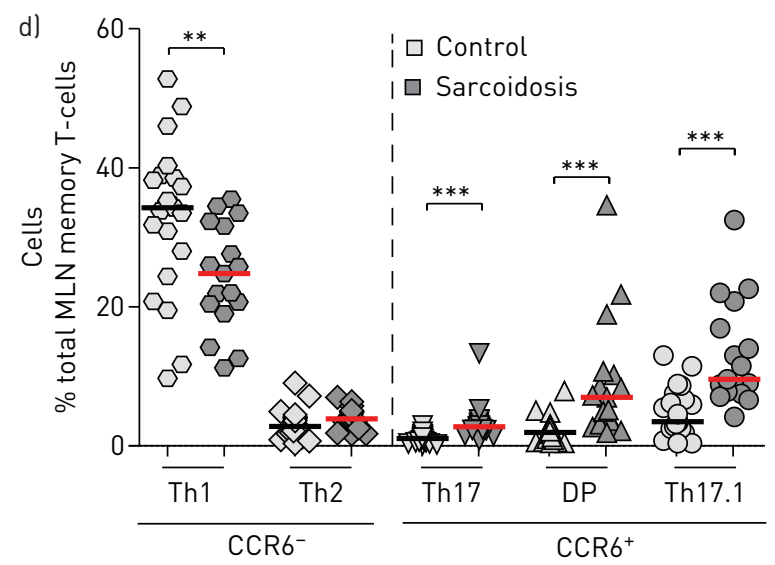

Sarcoidosis MLNs

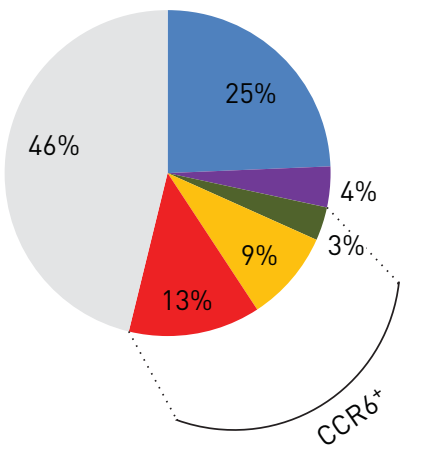

- Th1

- Th2

- Th17

DP

ㅁ Th17.1

Unclassified

FIGURE 1 Significant increase of T-helper (Th) 17.1 cells in sarcoidosis mediastinal lymph nodes (MLNs) compared with controls. CCR6 ${ }^{-}$T-cell subsets (CCR4 ${ }^{-} \mathrm{CXCR}^{+}$Th1 cells and $\mathrm{CCR}^{+} \mathrm{CXCR} 3^{-} \mathrm{Th}^{2}$ cells) and $\mathrm{CCR}^{+}{ }^{+}$T-cell subsets $\left(\mathrm{CCR} 4^{+} \mathrm{CXCR} 3^{-}\right.$Th17 cells, $\mathrm{CCR}^{+}{ }^{+} \mathrm{CXCR} 3^{+}$double-positive (DP) Th cells and CCR4 $^{-}$CXCR3 $^{+}$Th17.1 cells) were determined in CD45RA-FoxP3 low/int total memory CD4 $^{+}$T-cells $^{-}$(see supplementary figure E1 for gating strategy) from sarcoidosis bronchoalveolar lavage fluid (BALF) ( $\mathrm{n}=36$ ) and from control MLNs ( $n=22$ ) and sarcoidosis MLNs ( $n=17)$. a) Proportions of Th1, Th2, Th17, CCR6 ${ }^{+}$DP and Th17.1 cells of total memory $\mathrm{CD4}^{+} \mathrm{T}$-cells in sarcoidosis BALF. Horizontal lines indicate median values. Significance determined using the Wilcoxon signed-rank test. ***: $p \leqslant 0.001$ (sarcoidosis Th17.1 cells versus either sarcoidosis Th1, Th2 or Th17 or DP cells). b) Pie chart showing mean percentages of Th1, Th2, Th17, $\mathrm{CCR6}^{+} \mathrm{DP}$, Th17.1 and unclassified cells (which include CCR6 ${ }^{-} \mathrm{CCR}^{-}{ }^{-} \mathrm{CXCR} 3^{-}$cells, $\mathrm{CCR6}^{-} \mathrm{CCR}^{+}{ }^{+} \mathrm{CXCR} 3^{+} \mathrm{cells}^{-}$ and $\mathrm{CCR}^{+}{ }^{+} \mathrm{CCR} 4^{-} \mathrm{CXCR} 3^{-}$cells) of total memory $\mathrm{CD}^{+}{ }^{+}$-cells in sarcoidosis BALF. c) Proportions of CCR6 ${ }^{+}$ total memory T-cells in MLNs and d) proportions of Th1, Th2, Th17, CCR6 ${ }^{+}$DP and Th17.1 cells of total memory $\mathrm{CD}^{+} \mathrm{T}$-cells in MLNs. Horizontal lines indicate median values. Significance determined using the Mann-Whitney U-test. **: $\mathrm{p}<0.01{ }^{* * *}: \mathrm{p}<0.001$. e) Pie charts showing mean percentages of Th1, Th2, Th17, $\mathrm{CCR6}^{+} \mathrm{DP}$, Th17.1 and unclassified cells (which include CCR6 ${ }^{-} \mathrm{CCR}^{-}{ }^{-} \mathrm{CXCR}^{-}$cells, $\mathrm{CCR}^{-} \mathrm{CCR}^{+}{ }^{-} \mathrm{CXCR}{ }^{+} \mathrm{cells}^{-}$ and $\mathrm{CCR} 6^{+} \mathrm{CCR}^{-}{ }^{-} \mathrm{CXCR} 3^{-}$cells) of total memory $\mathrm{CD} 4^{+} \mathrm{T}$-cells in MLNs. 
were significantly decreased compared with controls, although Th1 cells remained (as in controls) the most prominent memory $\mathrm{T}$-cell population (figure $1 \mathrm{~d}$ and $\mathrm{e}$ ).

Taken together, these data show that sarcoidosis MLNs contain increased proportions of Th17.1 cells compared with controls, paralleling the lungs [5].

Th17.1 cells and CCR6 ${ }^{+}$double-positive cells are highly proliferative in sarcoidosis MLNs The increase of Th17.1 cells in sarcoidosis MLNs could be caused by enhanced proliferation; therefore, we assessed the proliferative status of T-cell subsets.

Proportions of proliferative $\left(\mathrm{Ki}-67^{+}\right) \mathrm{CD}^{+}$memory $\mathrm{T}$-cells were significantly increased in sarcoidosis MLNs compared with controls (figure 2a). Interestingly, specifically Th17.1 cells and CCR6 ${ }^{+}$ double-positive cells exhibited high fractions of proliferating Ki- $67^{+}$cells compared with controls (figure $2 \mathrm{~b}$ and c). This was particularly striking for the Th17.1 subpopulation, which in controls contained only very few Ki- $67^{+}$cells. However, CCR6 ${ }^{+}$double-positive cells contained the highest fraction of proliferating cells $(\sim 15 \%$, which was significantly higher than any of the other T-cell subsets) in sarcoidosis MLNs (figure 2b). Interestingly, CCR6 ${ }^{+}$double-positive cells also showed decreased CTLA4 expression in sarcoidosis MLNs compared with controls (figure 2d), as we have previously also shown for Th17 cells [19].

In summary, ex vivo phenotyping identified Th17.1 cells as a highly proliferative Th subset in MLNs of sarcoidosis patients compared with controls. Furthermore, $\mathrm{CCR}^{+}$double-positive cells also proliferate highly compared with other Th cell subsets in sarcoidosis MLNs.

Higher Th17.1 cell proportions in sarcoidosis MLNs and BALF than peripheral blood

Next, we aimed to quantify Th17-derived CCR6 ${ }^{+}$populations within sarcoidosis peripheral blood, MLNs and BALF.

Remarkably, in sarcoidosis patients, the proportions of memory Th cells that were CCR6 ${ }^{+}$were significantly higher in BALF $(\sim 75 \%)$ and MLNs $(\sim 31 \%)$ compared with peripheral blood $(\sim 19 \%)$ (supplementary figure E4). Th17 cell proportions were significantly lower in BALF than in MLNs and peripheral blood (figure 3a), whereas Th17.1 cells were significantly higher (figure 3c). Proportions of $\mathrm{CCR}^{+}$double-positive cells were similar in MLNs and BALF, but were significantly higher in BALF than peripheral blood (figure $3 \mathrm{~b}$ ). CCR6 ${ }^{-}$Th1 cell proportions were lower in sarcoidosis BALF compared with MLNs (supplementary figure E4). Importantly, paired analyses of the subgroup of patients that donated material from multiple organs (either peripheral blood and MLNs $(n=17)$ or peripheral blood and BALF $(\mathrm{n}=15))$ also showed a significant decrease in Th17 cells and a significant increase in Th17.1 cell proportions in MLNs or BALF compared with peripheral blood of the same patient (supplementary figure E5).

Taken together, the data show that Th17.1 cells are not a prominent population in the peripheral blood of sarcoidosis patients. Rather, patients show an increase in Th17.1 cells in MLNs, which is further enhanced in sarcoidosis lungs.

\section{Higher BALF Th17.1 cell proportions in patients developing chronic disease than in patients undergoing resolution}

As Th17.1 cells are described to be pathogenic and highly present in sarcoidosis lungs, we questioned whether proportions of Th17.1 cells in BALF would associate with disease prognosis. Therefore, we determined the disease course of a subgroup $(n=25)$ of our study cohort at $\sim 2$ years of clinical follow-up.

Interestingly, in patients developing chronic sarcoidosis, and specifically progressive chronic sarcoidosis with need for treatment, significantly higher BALF Th17.1 proportions were detected at time of diagnosis compared with patients undergoing resolution (figure 4a). In contrast, Th1 proportions were lower in patients developing progressive chronic disease than patients undergoing disease resolution (figure 4b), resulting in an increased Th17.1/Th1 ratio within BALF of patients developing chronic disease (figure 4c). Although nonsignificant, Th17 proportions also showed a trend to be lower in patients developing chronic disease than in patients undergoing resolution and patients developing progressive chronic disease showed a higher Th17.1/Th17 ratio within BALF (supplementary figure E6).

In conclusion, the data suggest that development of chronic disease is significantly associated with numbers of Th17.1 cells in sarcoidosis BALF, supporting a pathogenic role for these cells in the development of (chronic) pulmonary sarcoidosis.

\section{Discussion}

Our study is the first to show that Th17.1 cells are significantly increased in sarcoidosis MLNs compared with control MLNs, reflecting our previous findings in sarcoidosis lungs [5]. Furthermore, Th17.1 cell 

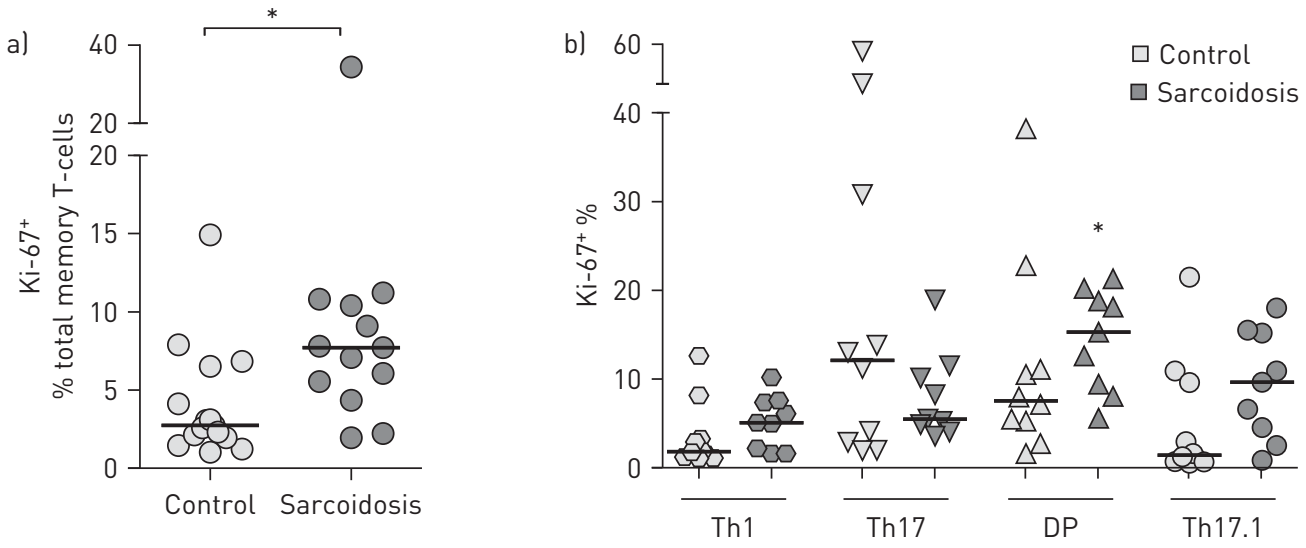

c)
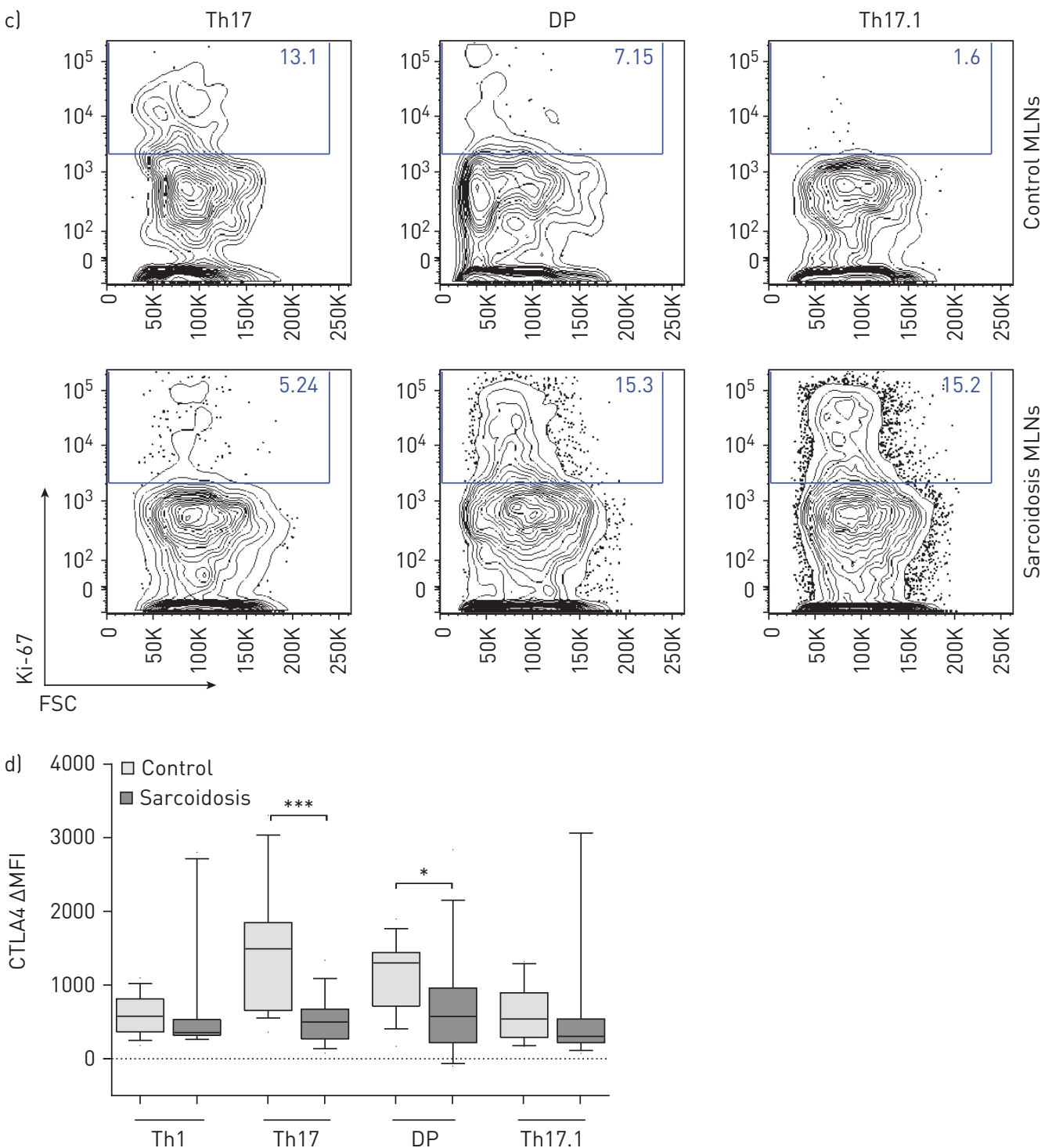

FIGURE 2 T-helper (Th) 17.1 cells and $\mathrm{CCR}^{+}$double-positive (DP) cells are highly proliferative in sarcoidosis mediastinal lymph nodes (MLNs). Expression of Ki-67 was determined ex vivo in Th1, Th17, CCR6 ${ }^{+}$DP and Th17.1 cells in control and sarcoidosis MLNs. a) Proportions of $\mathrm{Ki}-67^{+}$total memory T-cells (data from 15 controls and 13 patients). b) Proportions of $\mathrm{Ki}-67^{+}$cells in control $(n=10)$ and sarcoidosis MLNs ( $\left.n=9\right)$. Horizontal lines indicate median values. Significance in b) between median values of paired samples was determined using the Wilcoxon signed-rank test. *: $p<0.05$ (sarcoidosis DP cells versus either sarcoidosis Th1, Th17 or Th17.1). c) Representative flow cytometry analysis of Th17, CCR6 ${ }^{+}$DP and Th17.1 cells in MLNs from one control and one sarcoidosis patient. FSC: forward scatter. d) Change in mean fluorescence intensity ( $\triangle \mathrm{MFI}$ ) of cytotoxic T-lymphocyte antigen 4 (CTLA4) (data from 17 controls and 13 patients). Horizontal lines indicate median values. Significance determined using the Mann-Whitney U-test. *: $p<0.05 ;{ }^{* * *}: p<0.001$. 

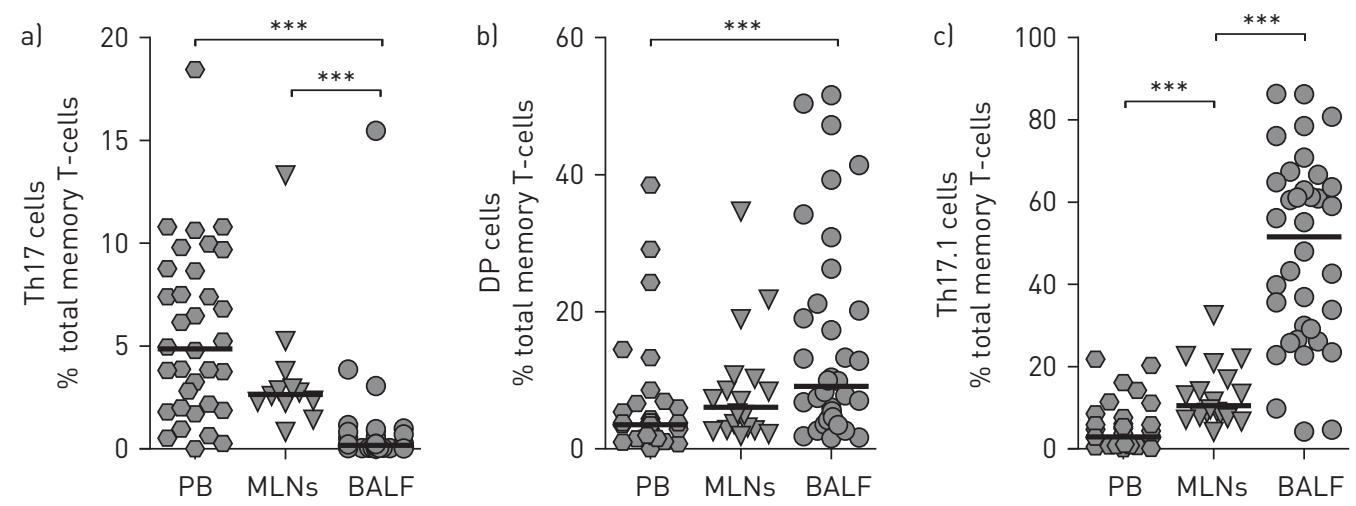

FIGURE 3 Higher T-helper (Th) 17.1 cell proportions in sarcoidosis mediastinal lymph nodes (MLNs) and bronchoalveolar lavage fluid (BALF) than peripheral blood (PB): proportions of a) Th17, b) CCR6 $^{+}$ double-positive (DP) and c) Th17.1 cells were determined in total memory $\mathrm{CD}^{+}$T-cells. Data from 34 (PB), 17 (MLNs) and 36 (BALF) patients. Horizontal lines indicate median values. Significance was determined using the Mann-Whitney U-test. ${ }^{* * *}: p<0.001$.

proportions are highest in the granulomatous BALF when compared with sarcoidosis-derived MLNs and peripheral blood. Higher proportions of Th17.1 cells in the lungs significantly correlated with development of chronic disease. Taken together, the data suggest that Th17.1 cell proportions in pulmonary sarcoidosis can be evaluated as a diagnostic and/or prognostic marker in clinical practice and could serve as a new therapeutic target.

Since it was recognised that Th17 cells display considerable plasticity and can produce IFN- $\gamma$, it has become increasingly clear that IFN- $\gamma$-producing Th17 cells play a key role in the development of autoimmune diseases $[17,20]$. IFN- $\gamma$-producing Th17 cells, or so-called Th17.1 cells, are described to be pathogenic in several autoimmune diseases and chronic inflammatory disorders, including Crohn's disease $[8,9]$ and arthritis [10]. In a previous study, we clarified that specifically CCR6 ${ }^{+}$Th17.1 cells rather than classical CCR6 ${ }^{-}$Th1 cells are the major source for IFN- $\gamma$ production in sarcoidosis BALF [5]. Interestingly, here, we found that in sarcoidosis MLNs not only Th17.1 cells, but all CCR6 ${ }^{+}$Th cell
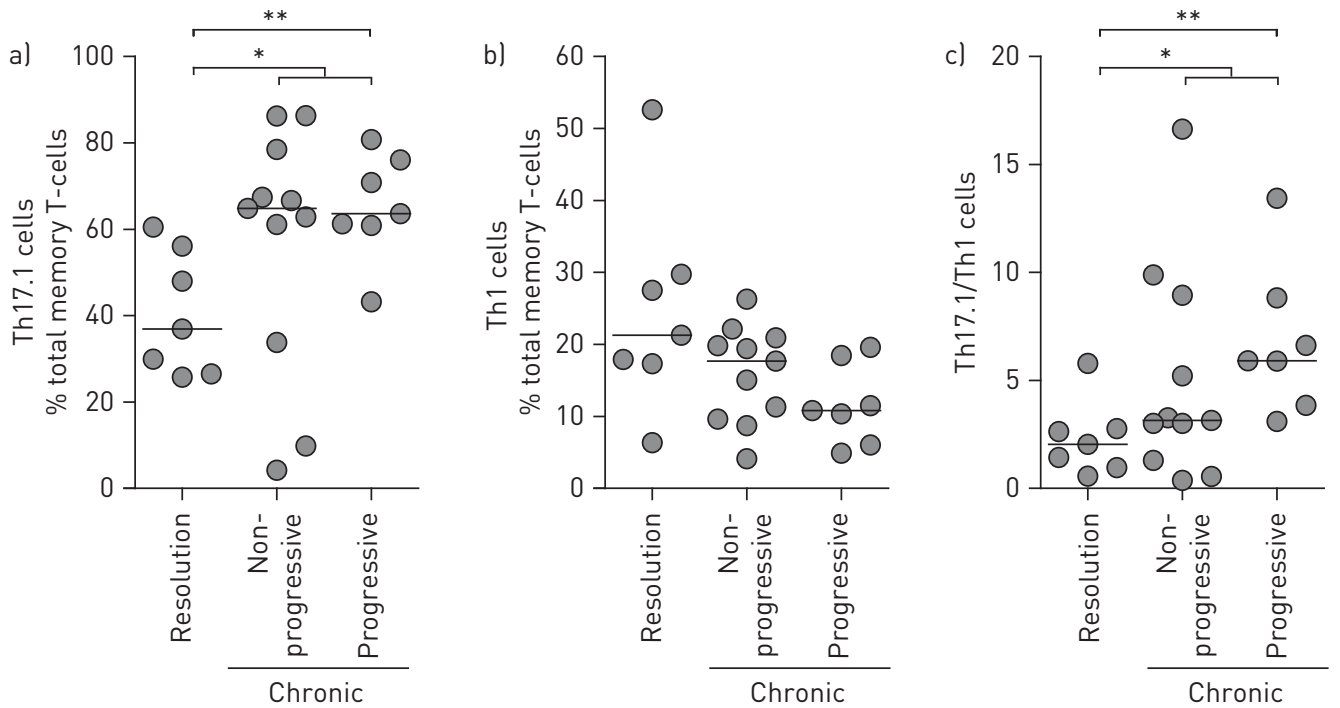

FIGURE 4 Higher bronchoalveolar lavage fluid T-helper (Th) 17.1 cell proportions in patients developing chronic disease than patients undergoing resolution. Disease course of a subgroup of patients (n=25) was determined 2 years after study inclusion and flow cytometric analyses. Resolution of disease ( $n=7)$ was defined by the absence of abnormalities on chest radiography and clinical symptoms. Patients with residual abnormalities on chest radiography but without need for treatment were designated as nonprogressive chronic $(n=11)$ and patients with need for treatment were designated as progressive chronic $(n=7)$. a) Proportion of Th17.1 cells and b) proportion of Th1 cells out of the total memory CD4 ${ }^{+} \mathrm{T}$-cells at time of diagnosis. c) Ratio of the proportions of Th17.1 versus Th1 cells. Horizontal lines indicate median values. Significance determined using the Mann-Whitney U-test. *: $p<0.05 ;{ }^{* *}: p<0.01$. 
subsets, including Th17 and CCR6 $^{+}$double-positive cells, were significantly enhanced compared with control MLNs. As Th17 and CCR6 ${ }^{+}$double-positive cells can be precursor cells of Th17.1 cells, the data may imply that initial Th17-lineage subset differentiation occurs within sarcoidosis MLNs [21].

In addition to supporting that Th17 cells are induced within sarcoidosis MLNs, our data suggest that chronically inflamed sites $[10,15]$ such as sarcoidosis lungs (but possibly also granulomatous parts of the MLNs) play a role in accelerating Th17.1 cell differentiation by inducing conversion of Th17 towards Th17.1 cells. For example, comparing different immunological compartments involved showed that specifically Th17.1 cells were augmented in sarcoidosis lungs compared with MLNs and peripheral blood. In joints of patients with juvenile idiopathic arthritis, Th17.1 cell fractions were also found increased compared with peripheral blood and shared clonal ancestry with Th17 cells [10]. Furthermore, although Th17.1 cells were abundantly present within sarcoidosis affected organs, we identified CCR6 ${ }^{+}$ double-positive cells, which were previously suggested to represent an intermediated stage between Th17 and Th17.1 cells [16-18], as the subset with the highest fraction of proliferating cells. Sarcoidosis CCR6 ${ }^{+}$ double-positive cells also showed decreased co-inhibitory CTLA4 expression compared with controls, likely contributing to increased proliferative capacity. Previously, we have also observed a reduced CTLA4 expression in sarcoidosis MLNs Th17 cells [19]. Aberrant expression of co-inhibitory receptors such as CTLA4 [19] on specific T-cell subsets in sarcoidosis is very likely to be part of disease pathogenesis, as clinical treatment with anti-CTLA4 has been associated with development of sarcoid-like granulomas in MLNs of cancer patients [19]. Finally, high levels of cytokines, such as interleukin (IL)-12 or IL-23, which can induce a Th17 shift towards Th17.1 cells, are found in sarcoidosis lungs [10, 13, 15, 22]. Increased protein expression of IL-12 (p70 and p40) is found consistently within sarcoidosis lungs [23-25]. Although IL-23p19 expression in sarcoid lungs remains uncertain, high expression of IL-12p40 compared with IL-12p70 may very well indicate elevated levels of IL-23 [25], as IL-12p40 is also a subunit of IL-23 [17]. Notably, IL-23 transcription was enhanced in sarcoid skin lesions compared with controls [26] and serum amyloid $\mathrm{A}$, an antigen that was speculated to contribute to the development of chronic (pulmonary) sarcoidosis [27], has been described to increase IL-23 production by dendritic cells [28]. Strikingly, BALF IL-12p40 protein levels are highest in patients developing chronic disease [29]. Our data cannot exclude the possibility of additional selective Th17.1 cell migration towards sarcoidosis lungs [15], attracted by high local coexpression of IFN- $\gamma$-induced protein 10/CXCL10 [30] and CCL20 [31], ligands for CXCR3. Nevertheless, Th1 cell attraction towards sarcoidosis lungs would then also be expected as these cells also express CXCR3 and we did not find evidence for this phenomenon.

Highly clinically relevant, we found significantly higher Th17.1 cell proportions at the time of diagnosis in lungs of patients who develop chronic disease compared with patients who underwent resolution. Together with our previous finding of increased BALF Th17.1 cell proportions in a population that included progressive patients who were on first-, second- and/or third-line therapy [5], these data strongly argue for a pathogenic role for Th17.1 cells in the development/progression of pulmonary sarcoidosis. Remarkably, two independent single nucleotide polymorphisms within the IL23R gene locus were found to predispose for (chronic) sarcoidosis [32, 33] and IL-23 receptor (IL-23R) expression is essential for conversion of Th17 cells towards pathogenic Th17.1 cells during chronic inflammation in mice $[13,34]$. The most recent genome-wide association study identified one single nucleotide polymorphism within the putative promoter region of the IL23R gene, which might influence IL-23R expression [33]. The functional prediction and protein network analyses of the identified candidate susceptibility genes provided a prominent role for the IL-23/Th17 signalling pathway in the genetic aetiology of sarcoidosis [33]. Together with our data, this implies that genetic variations within the IL23R gene or other genes involved in the IL-23/Th17 signalling pathway may predispose for (chronic) sarcoidosis by supporting the conversion of Th17 to Th17.1 cells or by stabilising Th17.1 cells. Therefore, specific targeting of IL-23R signalling, e.g. by tildrakizumab/guselkumab (anti-IL-23p19), which was recently described to be successful in psoriasis [35], might be a promising new therapeutic approach in (chronic) sarcoidosis.

Additional prospective studies investigating $\mathrm{CCR}^{+}$Th17-lineage cell function, such as cytokine production, in sarcoidosis patients with a distinct disease prognosis are needed to address the functional complexity of Th17-lineage cells. This is highlighted by recently reported findings in Löfgren's disease [36], a subtype of acute sarcoidosis whereby patients are unlikely to develop chronic disease. In these patients, lung Th17.1 cells were associated with a favourable disease outcome [36]. Löfgren BALF contained higher levels of favourable Th17 cytokines such as IL-17A, IL-10 and IL-2, likely contributing to the favourable prognosis in Löfgren patients; however, non-Löfgren sarcoidosis patients have higher levels of IFN- $\gamma$ compared with Löfgren patients, possibly contributing to a worse outcome [36]. Furthermore, Löfgren syndrome is associated with a highly inflammatory profile during active disease, which would confirm the pathogenic potential of Th17.1 cells. 
In conclusion, this study shows that sarcoidosis CCR6 ${ }^{+}$Th17-lineage cells are significantly increased in granulomatous MLNs and likely differentiate into Th17.1 cells in the lung microenvironment, where their presence significantly correlates with development of chronic disease. This study provides a new perspective on 1) sarcoidosis pathogenesis, 2) the value of Th17.1 cells as a potential diagnostic/prognostic marker and 3) the potential of new therapeutic targets.

\section{Acknowledgements}

The authors gratefully acknowledge patients, research nurses and physicians participating in this study from Erasmus MC, Franciscus Gasthuis \& Vlietland, Leiden University Medical Center and Ikazia hospital in the Netherlands. The authors thank Ingrid Bergen and Jennifer van Hulst (Erasmus MC, Rotterdam, The Netherlands) for technical assistance, and Erik Lubberts (Erasmus MC, Rotterdam, The Netherlands) for critical discussions.

Author contributions: C.E. Broos, M. van Nimwegen, S.M.J. Paulissen, J.C.C.M. in 't Veen, H.C. Hoogsteden, R.W. Hendriks, B. van den Blink and M. Kool designed the study and the experiments. C.E. Broos, M. van Nimwegen, J.C.C.M. in 't Veen, S.M.J. Paulissen, J.P. van Hamburg, J.T. Annema, R. Heller-Baan, A. Kleinjan and M.S. Wijsenbeek were responsible for flow cytometry and data collection. C.E. Broos, L.L. Koth, R.W. Hendriks, B. van den Blink, M. Kool analysed the data. C.E. Broos, L.L. Koth, M.S. Wijsenbeek, R.W. Hendriks, B. van den Blink and M. Kool drafted the manuscript. All authors read, critically revised and approved the final manuscript.

\section{References}

1 Statement on sarcoidosis. Joint Statement of the American Thoracic Society (ATS), the European Respiratory Society (ERS) and the World Association of Sarcoidosis and Other Granulomatous Disorders (WASOG) adopted by the ATS Board of Directors and by the ERS Executive Committee, February 1999. Am J Respir Crit Care Med 1999; 160: 736-755.

2 Arkema EV, Grunewald J, Kullberg S, et al. Sarcoidosis incidence and prevalence: a nationwide register-based assessment in Sweden. Eur Respir J 2016; 48: 1680-1699.

3 Baughman RP, Field S, Costabel U, et al. Sarcoidosis in America. Analysis based on health care use. Ann Am Thorac Soc 2016; 13: 1244-1252.

4 Baughman RP, Grutters JC. New treatment strategies for pulmonary sarcoidosis: antimetabolites, biological drugs, and other treatment approaches. Lancet Respir Med 2015; 3: 813-822.

5 Ramstein J, Broos CE, Simpson LJ, et al. Interferon-gamma-producing Th17.1 cells are increased in sarcoidosis and more prevalent than Th1 cells. Am J Respir Crit Care Med 2015; 193: 1281-1291.

6 von Bartheld MB, Dekkers OM, Szlubowski A, et al. Endosonography vs conventional bronchoscopy for the diagnosis of sarcoidosis: the GRANULOMA randomized clinical trial. JAMA 2013; 309: 2457-2464.

7 Ten Berge B, Paats MS, Bergen IM, et al. Increased IL-17A expression in granulomas and in circulating memory T cells in sarcoidosis. Rheumatology 2012; 51: 37-46.

8 Annunziato F, Cosmi L, Santarlasci V, et al. Phenotypic and functional features of human Th17 cells. J Exp Med 2007; 204: 1849-1861.

9 Ramesh R, Kozhaya L, McKevitt K, et al. Pro-inflammatory human Th17 cells selectively express P-glycoprotein and are refractory to glucocorticoids. J Exp Med 2014; 211: 89-104.

10 Nistala K, Adams S, Cambrook H, et al. Th17 plasticity in human autoimmune arthritis is driven by the inflammatory environment. Proc Natl Acad Sci USA 2010; 107: 14751-14756.

11 Acosta-Rodriguez EV, Rivino L, Geginat J, et al. Surface phenotype and antigenic specificity of human interleukin 17-producing T helper memory cells. Nat Immunol 2007; 8: 639-646.

12 Boniface $\mathrm{K}$, Blumenschein WM, Brovont-Porth $\mathrm{K}$, et al. Human Th17 cells comprise heterogeneous subsets including IFN-gamma-producing cells with distinct properties from the Th1 lineage. J Immunol 2010; 185: 679-687.

13 Hirota K, Duarte JH, Veldhoen M, et al. Fate mapping of IL-17-producing T cells in inflammatory responses. Nat Immunol 2011; 12: 255-263.

14 Maggi L, Santarlasci V, Capone M, et al. Distinctive features of classic and nonclassic (Th17 derived) human Th1 cells. Eur J Immunol 2012; 42: 3180-3188

15 Sundrud MS, Trivigno C. Identity crisis of Th17 cells: many forms, many functions, many questions. Semin Immunol 2013; 25: 263-272.

16 Paulissen SM, van Hamburg JP, Dankers W, et al. The role and modulation of CCR6 ${ }^{+}$Th17 cell populations in rheumatoid arthritis. Cytokine 2015; 74: 43-53.

17 Lubberts E. The IL-23-IL-17 axis in inflammatory arthritis. Nat Rev Rheumatol 2015; 11: 415-429.

18 Paulissen SM, van Hamburg JP, Davelaar N, et al. CCR6 ${ }^{+}$Th cell populations distinguish ACPA positive from ACPA negative rheumatoid arthritis. Arthritis Res Ther 2015; 17: 344.

19 Broos CE, van Nimwegen M, in 't Veen JCCM, et al. Decreased cytotoxic T-lymphocyte antigen 4 expression on regulatory T cells and Th17 cells in sarcoidosis: double trouble? Am J Respir Crit Care Med 2015; 192: 763-765.

20 Basu R, Hatton RD, Weaver CT. The Th17 family: flexibility follows function. Immunol Rev 2013; 252: 89-103.

21 Broos CE, van Nimwegen M, Hoogsteden HC, et al. Granuloma formation in pulmonary sarcoidosis. Front Immunol 2013; 4: 437.

22 Ghoreschi K, Laurence A, Yang XP, et al. Generation of pathogenic $\mathrm{T}_{\mathrm{H}} 17$ cells in the absence of TGF-beta signalling. Nature 2010; 467: 967-971.

23 Moller DR, Forman JD, Liu MC, et al. Enhanced expression of IL-12 associated with Th1 cytokine profiles in active pulmonary sarcoidosis. J Immunol 1996; 156: 4952-4960.

24 Shigehara K, Shijubo N, Ohmichi M, et al. IL-12 and IL-18 are increased and stimulate IFN-gamma production in sarcoid lungs. J Immunol 2001; 166: 642-649.

25 Idali $\mathrm{F}$, Wiken $\mathrm{M}$, Wahlström J, et al. Reduced Th1 response in the lungs of HLA-DRB1*0301 patients with pulmonary sarcoidosis. Eur Respir J 2006; 27: 451-459. 
Judson MA, Marchell RM, Mascelli M, et al. Molecular profiling and gene expression analysis in cutaneous sarcoidosis: the role of interleukin-12, interleukin-23, and the T-helper 17 pathway. J Am Acad Dermatol 2012; 66: 901-910.

27 Chen ES, Moller DR. Etiologic role of infectious agents. Semin Respir Crit Care Med 2014; 35: $285-295$.

28 Ather JL, Ckless K, Martin R, et al. Serum amyloid A activates the NLRP3 inflammasome and promotes Th17 allergic asthma in mice. J Immunol 2011; 187: 64-73.

29 Barbarin V, Petrek M, Kolek V, et al. Characterization of p40 and IL-10 in the BALF of patients with pulmonary sarcoidosis. J Interferon Cytokine Res 2003; 23: 449-456.

30 Agostini C, Cassatella M, Zambello R, et al. Involvement of the IP-10 chemokine in sarcoid granulomatous reactions. J Immunol 1998; 161: 6413-6420.

31 Facco M, Baesso I, Miorin M, et al. Expression and role of CCR6/CCL20 chemokine axis in pulmonary sarcoidosis. J Leukoc Biol 2007; 82: 946-955.

32 Fischer A, Nothnagel M, Franke A, et al. Association of inflammatory bowel disease risk loci with sarcoidosis, and its acute and chronic subphenotypes. Eur Respir J 2011; 37: 610-616.

33 Fischer A, Ellinghaus D, Nutsua M, et al. Identification of immune-relevant factors conferring sarcoidosis genetic risk. Am J Respir Crit Care Med 2015; 192: 727-736.

34 Awasthi A, Riol-Blanco L, Jager A, et al. Cutting edge: IL-23 receptor GFP reporter mice reveal distinct populations of IL-17-producing cells. J Immunol 2009; 182: 5904-5908.

35 Gordon KB, Duffin KC, Bissonnette R, et al. A phase 2 trial of guselkumab versus adalimumab for plaque psoriasis. N Engl J Med 2015; 373: 136-144.

36 Kaiser Y, Lepzien R, Kullberg S, et al. Expanded lung T-bet ${ }^{+} \mathrm{ROR} \gamma \mathrm{T}^{+} \mathrm{CD} 4^{+} \mathrm{T}$-cells in sarcoidosis patients with a favourable disease phenotype. Eur Respir J 2016; 48: 484-494. 